\title{
Recurrent Dedifferentiated Chordoma
}

National Cancer Institute

\section{Source}

National Cancer Institute. Recurrent Dedifferentiated Chordoma. NCI Thesaurus. Code C162728.

The reemergence of dedifferentiated chordoma after a period of remission. 